\title{
Measurements of the Relaxation Kinetics of Photo-Excited Enol Form of Dibenzoylmethane
}

\author{
P. D. YANKOV, S. M. SALTIEL and I. V. TOMOV
}

Faculty of Physics, Sofia University, BG-1126, Sofia, Bulgaria

P. J. MARKOV and I. K. PETKOV

Department of Organic Chemistry, University of Sofia, BG-1126, Bulgaria

(Received 29 March, 1984; Revised manuscript accepted 6 June, 1984)

The decay rate of the excited state of the enol form of dibenzoylmethane (DBM) in different solvents is measured directly using nanosecond light pulses. The observed relaxation kinetics is explained by the formation of three intermediate forms of the excited cis-enol form of DBM. The decay rates of the intermediates and their absorption cross sections for $\lambda=266 \mathrm{~nm}$ are reported too.

\section{INTRODUCTION}

The photochemical behaviour of enolizable $\beta$-dicarbonyl compounds, their derivatives and analogous have been a subject of recent intensive studies. As a result of a photoinduced hydrogen transfer, the photoactive enol-form turns into the keto tautomer<smiles>[R]C(=O)C([2H])C([R])=O</smiles>

Experimental ${ }^{1-3}$ and theoretical ${ }^{4-6}$ data give evidence to suggest that excitation of the chelated enol form leads to the formation of more 
than one non-chelated short-lived species. We are reporting here the results of the determination of the decay time of the excited dibenzoylmethane enol form and the probable energy scheme of the relaxation process.

\section{EXPERIMENTAL}

\section{Apparatus}

The experimental set-up is shown in Figure 1. It consists of two synchronized Nd:YAG lasers A and B. The laser A described in detail elsewhere, ${ }^{7}$ produced light pulses with duration of $5 \mathrm{~ns}$ at $\lambda=1.06 \mu \mathrm{m}$. The laser B was Q-switched to produce pulses of $7 \mathrm{~ns}$ duration at the same wavelength. The lasers operated with a pulse repetition rate up to $10 \mathrm{~Hz}$ in $\mathrm{TEM}_{00}$ mode. The delay time between the output pulses of both lasers was varied in the range $0-10 \mathrm{~s}$ by a proper electronic control of the electrooptic switches used in both lasers. The jitter of the delay time was $10 \mathrm{~ns}$ within the range of $0-1 \mu \mathrm{s}$ and less than $1 \%$ within the range of $1 \mu s-10 \mathrm{~s}$.

The output radiation of the Nd:YAG lasers was up-converted in the UV region by nonlinear crystals CDA and KDP. The third harmonic of laser A with pulse energy of $E_{3}=2 \mathrm{~mJ}$ was used as an exciting

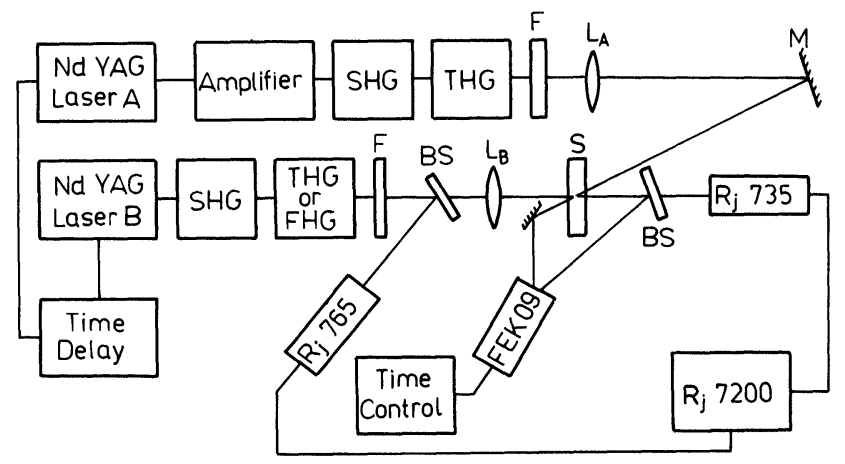

FIGURE 1 Experimental set up. SHG, THG, FHG-nonlinear crystals for second, third and fourth harmonic generation, $F$-filters, $L_{A}$ and $L_{B}$ - quartz lenses, $B S$-beam splitters, $\boldsymbol{M}$-mirror. 
source. The third or forth harmonic $\left(E_{3}=0.04 \mathrm{~mJ}, \lambda_{p}=355 \mathrm{~nm} ; E_{4}=\right.$ $0.01 \mathrm{~mJ}, \lambda_{p}=266 \mathrm{~nm}$ ) of the laser B was used as a probe pulse. The exciting light was focused in the sample $S$ by a $1 \mathrm{~m}$ focal length quartz lens $L_{A}$. The probe light pulse was focused by a $13 \mathrm{~cm}$ focal length quartz lens $L_{B}$. The solution thickness in the sample cell was $5 \mathrm{~mm}$. The angle between the exciting and probe beams was chosen to be $4^{\circ}$ in order to obtain good overlapping of both beams in the sample. By propagating the exciting and probe beams in opposite directions a $S / N$ ratio of $10^{2}$ was achieved. The pulse energies were measured with Energy Ratiometer $R_{j} 7200$. The delay time between the pulses was monitored by FEK 09 vacuum photodiode and Tektronix 466 oscilloscope.

The appropriate energies of the exciting and probe pulses were determined after studying the nonlinear absorption of the enol form of DBM (ca. $1 \times 10^{-4} \mathrm{~mol} \cdot 1^{-1}$ ). It was found that the absorption did not depend on the energy density of the radiation when it was below $0.1 \mathrm{~J} \cdot \mathrm{cm}^{-2}$ (i.e., the range of linear absorption) and that the saturation of the absorption took place for energy densities above $1.5 \mathrm{~J} \cdot \mathrm{cm}^{-2}$. During the measurements the energy densities of the probe and exciting pulses were chosen to be within these two ranges, respectively.

\section{Solutions}

Dibenzoylmethane was "analytical purity" Merck commercial product. Isooctane, cyclohexane and methanol (Merck "spectroscopic grade") were used as solvents. Solutions with concentration of $1 \times$ $10^{-4} \mathrm{~mol} \cdot \mathrm{l}^{-1}$ were prepared and kept in the dark until thermodynamic equilibrium between two tautomeric forms was established.

\section{Irradiation}

The wavelength of the exciting pulse $\lambda_{e}=355 \mathrm{~nm}$ was chosen to be in the absorption band of the enol dibenzoylmethane form (Figure 2). On the other hand the wavelength of the probe was either the same $355 \mathrm{~nm}$ or $266 \mathrm{~nm}$. The wavelength of $266 \mathrm{~nm}$ is close to the absorption maximum of one of the assumed transient form of DBM according to Ref. 2. 


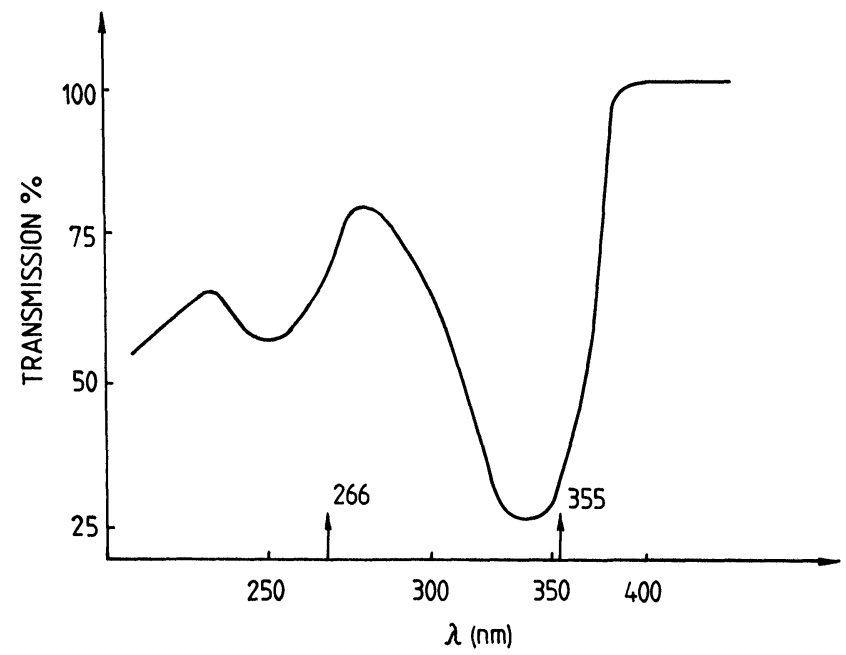

FIGURE 2 UV transmission spectra of DBM in isooctane solution $\left(1 \times 10^{-14} \mathrm{~mol} \cdot 1^{-1}\right.$, $l=2 \mathrm{~mm}$ ). Positions of the third and fourth harmonic are indicated. Note that the absorption band near $250 \mathrm{~nm}$ is due to the keto form of DBM which exists as a result of thermodynamic equilibrium between the two tautomeric forms.

\section{EXPERIMENTAL RESULTS}

The probe pulse transmission through the sample with isooctane solution of DBM as a function of delay time between the exciting and probe pulse is shown in Figure $3 \mathrm{a}\left(\lambda_{p}=355 \mathrm{~nm}\right)$ and Figure $3 \mathrm{~b}\left(\lambda_{p}=\right.$ $266 \mathrm{~nm}$ ). As it is seen while the transmission at $355 \mathrm{~nm}$ decreases to its initial (before irradiation) value, the transmission at $266 \mathrm{~nm}$ keeps the reverse trend.

We tried to match the experimental results (dots on Figure 3) with an analytical expression which involves a sum of exponential terms. It was found that a sum of three exponential terms produces a good fit. Therefore the normalized transmission may be presented as:

$$
\begin{aligned}
\ln \left[T_{3}(t) / T_{3}(\infty)\right] & =\sum_{i=1}^{3} A_{i 3} \exp \left(-q_{i 3} t\right) \\
-\ln \left[T_{4}(t) / T_{4}(\infty)\right] & =\sum_{i=1}^{3} A_{i 4} \exp \left(-q_{i 4} t\right)
\end{aligned}
$$




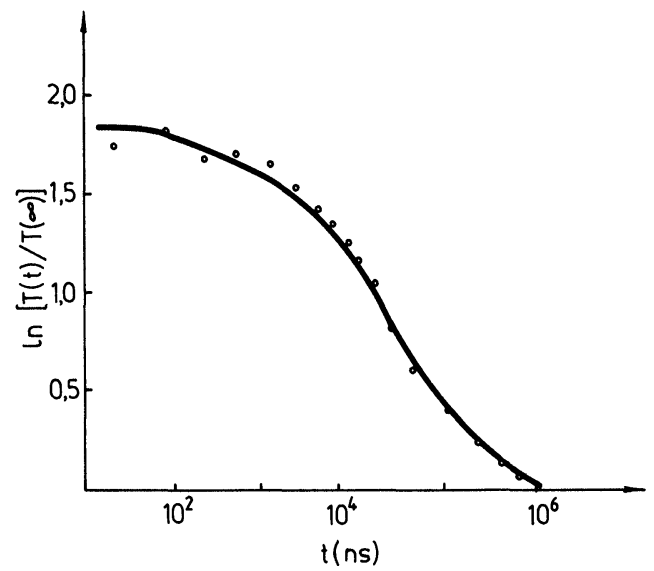

FIGURE 3a Transmission of probe pulse with $\lambda_{p}=355 \mathrm{~nm}$ versus delay time after exciting pulse with $\lambda_{e}=355 \mathrm{~nm}$.

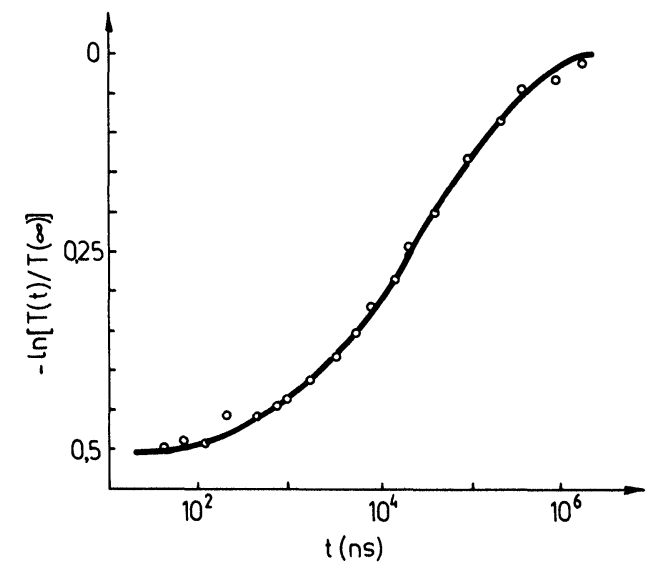

FIGURE $3 \mathrm{~b}$ Transmission of probe pulse with $\lambda_{p}=266 \mathrm{~nm}$ versus delay time after exciting pulse with $\lambda_{e}=355 \mathrm{~nm}$.

Here the subscrit " 3 " refers to the experiments with the third harmonic probe pulse $\lambda_{p}=355 \mathrm{~nm}$ and " 4 " to the forth harmonic probe pulse $\lambda_{p}=266 \mathrm{~nm} . T_{3}(\infty)$ and $T_{4}(\infty)$ are probe pulse transmissions without the exciting pulse.

The values of the corresponding coefficients $A_{i j}$ and $q_{i j}$ are presented in Table I. Note that, to within the experimental error the obtained 


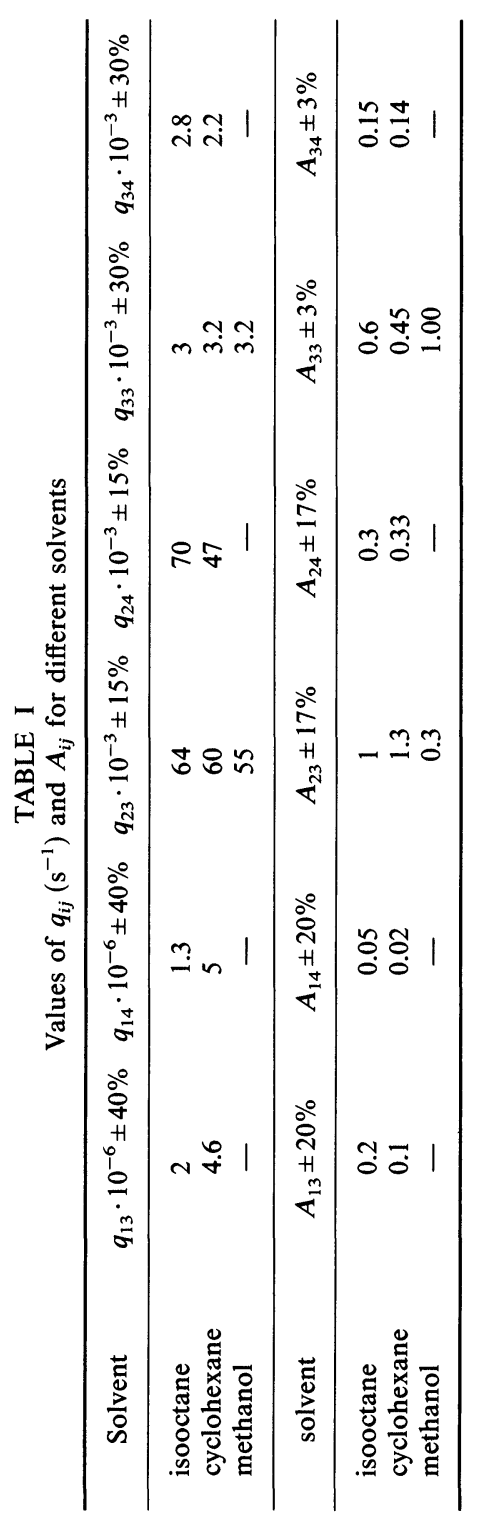


values of $q_{i 3}$ and $q_{i 4}$ are similar for each solvent. The solid curves in Figure 3 are calculated from Eq. (2) with the coefficients from Table I. Thus the observed relaxation processes are described fairly well by the "three exponential law."

Due to the very low value of $\ln \left[T_{4}(\infty) / T_{4}(t)\right],(S / N \sim 1)$, in methanol solution, the accuracy of $q_{i 4}$ and $A_{i 4}$ values were not acceptable and they are not included in Table I. $A_{13}$ and $q_{13}$ could not be determined for the same reason.

\section{MODEL AND DISCUSSION}

Before presenting a model of the relaxation process we point out three effects which may influence the transmission process but should be excluded due to their slow recovery time. These three effects are formation of keto tautomer molecules, diffusion and thermal effects. First, the formation of keto tautomer molecules during the exciting pulse should be excluded since the reverse process keto-enol is very slow $^{8}$ and takes several hours. Second, one may assume that the excited molecules leave irradiated volume due to diffusion. However, our estimation has shown that the diffusion from a beam waist of $100 \mu \mathrm{m}$ diameter is important in a time scale of about 1-2 seconds. Third, the study of far field beam divergence did not indicate any beam distortion due to thermal effects or microbubbles. Note that, in the experiment $1 \mathrm{~ms}$ after the exciting pulse, the transmission of both probe pulses $\left(\lambda_{p 3}\right.$ or $\left.\lambda_{p 4}\right)$ is practically the same as before the excitation.

Now we consider the experiment in which a probe pulse with $\lambda_{p}=355 \mathrm{~nm}$ was used (Figure $3 \mathrm{a}$ ). The lifetime of the first excited state $S_{1}$ is too small $\left(10^{-10}-10^{-9} \mathrm{~s}\right),{ }^{5}$ in comparison to the time resolution in this experiment, therefore we may assume in our model that immediately after the end of the exciting pulse $S_{1}$ is empty and the molecules occupy the lower levels. In this case the probe pulse transmission is determined by the number of molecules at the ground level $S_{0}$. Since the three exponential law fits very well the experimental results we can assume that there are three levels situated between ground state $S_{0}$ and first excited state $S_{1}$. The molecules which occupy these three excited levels are returning back to $S_{0}$ level with different rates. 
According to the given assumptions above, an energy level scheme shown in Figure 4 can be proposed. After the end of the exciting pulse the total number of molecules $N$ are distributed among the levels one to four:

$$
N=\sum_{i=1}^{4} n_{i}(t)
$$

where $n_{i}(t)$ is number of molecules on level $i$. For this four level system we write the rate equations:

$$
\begin{aligned}
& \frac{d n_{2}}{d t}=-k_{21} n_{2} \\
& \frac{d n_{3}}{d t}=-\left(k_{31}+k_{34}\right) \cdot n_{3} \\
& \frac{d n_{4}}{d t}=k_{34} n_{3}-k_{41} n_{4} \\
& \frac{d n_{1}}{d t}=k_{21} n_{2}+k_{31} n_{3}+k_{41} n_{4}
\end{aligned}
$$

The solution of the system gives:

$$
\begin{aligned}
& n_{2}=N_{2} \cdot \exp \left(-k_{21} t\right) \\
& n_{3}=N_{3} \cdot \exp (-k t) \\
& n_{4}=C_{1}\left\{\exp \left(-k_{41} t\right)-\exp \left[-\left(k_{21}+k_{34}\right) t\right]\right\} \\
& n_{1}=N-N_{2} \cdot \exp \left(-k_{21} t\right)-C_{2} \cdot \exp (-k t)-C_{1} \cdot \exp \left(-k_{41} t\right)
\end{aligned}
$$

where:

$$
\begin{aligned}
k & =k_{31}+k_{34} \\
C_{1} & =N_{3} k_{34} /\left(k-k_{41}\right) \\
C_{2} & =N_{3}\left(k_{31}-k_{41}\right) /\left(k-k_{41}\right)
\end{aligned}
$$

Here $N_{2}$ and $N_{3}$ are numbers of the particles on the levels 2 and 3 at the moment immediately following the exciting pulse, i.e., when delay time is $t=0$.

On the other hand:

$$
\ln \left[T_{3}(t) / T_{3}(\infty)\right]=\left[N-n_{1}(t)\right] \sigma_{3} l
$$




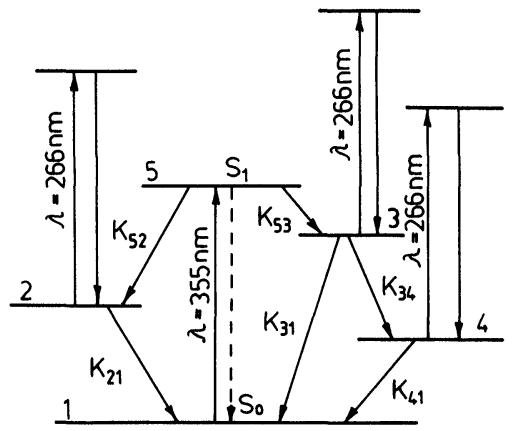

FIGURE 4 Energy diagram: $k_{i j}$-rate of population of $j$ level from $i$ level.

Here $\sigma_{3}$ is the absorption cross section for $\lambda_{p}=355 \mathrm{~nm}$ and $l$ is the cell thickness.

Substitution of (11) in (12) gives:

$$
\begin{aligned}
\ln [ & \left.T_{3}(t) / T_{3}(\infty)\right] \\
& =\sigma_{3} l\left[N_{2} \cdot \exp \left(-k_{21} t\right)+C_{1} \cdot \exp \left(-k_{41} t\right)+C_{2} \cdot \exp (-k t)\right]
\end{aligned}
$$

After comparing (13) and (2a) we find that

$$
A_{13}=\sigma_{3} l N_{2} \quad A_{23}=\sigma_{3} l C_{2} \quad A_{33}=\sigma_{3} l C_{1}
$$

and

$$
q_{13}=k_{21} \quad q_{23}=k_{31}+k_{34} \quad q_{33}=k_{41}
$$

The experimentally derived coefficients $A_{i 3}$ and $q_{i 3}$ permit the values of $k_{31}, k_{34}$ and their ratio $k_{31} / k_{34}$ to be determined. For isooctane solution the ratio $k_{31} / k_{34}$ is $2 \mp 0.9$, for cyclohexane $k_{31} / k_{34}=3 \mp 1.3$ and for methanol $k_{31} / k_{34}=0.35 \mp 0.16$.

In order to explain the second part of the experiment, in which a probe pulse was $\lambda_{p}=266 \mathrm{~nm}$, we use the same energy diagram (Figure 4) and the same rate equations (4-7). As non-excited enol form of DBM has a small absorption at this wavelength (see Figure 2 and note under it) only the levels 2,3 and 4 are contributing to the 
absorption of radiation with $\lambda_{p}=266 \mathrm{~nm}$. Then:

$$
T_{4}(t)=T_{4}(\infty) \sum_{i=2}^{4} \exp \left(n_{i} \sigma_{i 4} l\right)
$$

where $\sigma_{i 4}$ is the absorption cross-section for $\lambda_{p}=266 \mathrm{~nm}$ from $i$ level.

After substituting $n_{i}$ from (8), (9) and (10) in (16) we obtain the following expression:

$$
\begin{aligned}
\ln \left[T_{4}(\infty) / T(t)\right]= & l\left[N_{2} \sigma_{24} \cdot \exp \left(-k_{21} t\right)+C_{1} \sigma_{44} \cdot \exp \left(-k_{41} t\right)\right. \\
& \left.+\left(N_{3} \sigma_{34}-\sigma_{44} C_{1}\right) \cdot \exp (-k t)\right]
\end{aligned}
$$

We compare this expression with eq. (2b) and we find

$$
A_{14}=N_{2} \sigma_{24} l \quad A_{24}=\left(N_{3} \sigma_{34}-\sigma_{44} C_{1}\right) l \quad A_{34}=C_{1} \sigma_{44} l
$$

and

$$
q_{14}=k_{21} \quad q_{24}=k_{31}+k_{34} \quad q_{34}=k_{41}
$$

Thus our model gives equal values of $q_{i 3}$ and $q_{i 4}$ as it was found from the experimental data, and this supports the chosen model.

The values of the absorption cross sections $\sigma_{24}, \sigma_{34}$ and $\sigma_{44}$ at $\lambda_{p}=266 \mathrm{~nm}$ were also determined and are presented in Table II.

Now we try to associate the energy states shown in Figure 4 with possible transformations of the chelated enol form of the DBM molecule (Scheme 1e). PPP calculations [9] show that when the molecule is at $S_{1}$ state the 2-3 bond order decreases and has nearly the same magnitude as the $3-4$ bond. This means that a rotation round the $2-3$ bond is highly probable. On the other hand, the quantum chemical calculations ${ }^{5}$ of the energies of $S_{1}$ and $T_{1}$ states indicate that these energies become equal at $90^{\circ}$ rotation round the $2-3$ bond (Scheme 1b). Because of this there is a fast intersystem crossing

TABLE II

Values of absorption cross-sections $\sigma_{i 4}\left(\mathrm{~cm}^{2}\right)$ for $\lambda_{p}=266 \mathrm{~nm}$ for different solvents

\begin{tabular}{lccc}
\hline \multicolumn{1}{c}{ Solvent } & $\sigma_{24} \cdot 10^{14}$ & $\sigma_{34} \cdot 10^{14}$ & $\sigma_{44} \cdot 10^{14}$ \\
\hline is.ooctane & 1.6 & 1.9 & 2.8 \\
cyclohexane & 0.9 & 1.7 & 2.8 \\
methanol & - & 0.9 & 1.0 \\
\hline
\end{tabular}




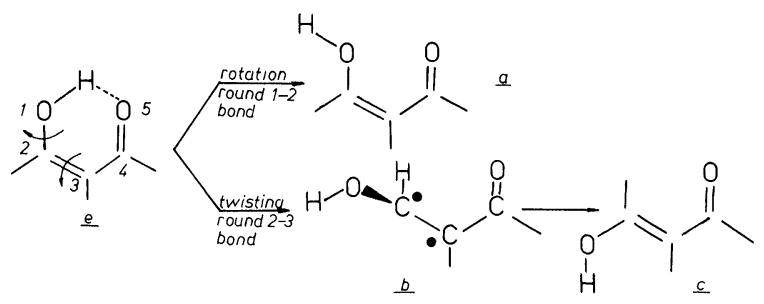

SCHEME 1

$S_{1} \rightarrow T_{1}$ which corresponds to the transition $5 \rightarrow 3$ (Figure 4). Arguments for formation of orthogonal triplet of the ethyl acetoacetate enol form have recently been presented by Gefoff $e t$ al. ${ }^{10}$ An additional rotation round the same bond leads to formation of a nonchelated isomer of the enol form (c) and we relate this transformation to the transition $3 \rightarrow 4$ in the energy diagram.

Another possibility for relaxation of the excited molecules is formation of a nonstable rotamer (Scheme 1a) formed by rotation round 1-2 bond. The possibility of this rotation has been discussed elsewhere. ${ }^{4}$ This rotamer may be related with the state 2 in the energy diagram.

In conclusion, using nanosecond pulses we were able to study the relaxation of DBM excited enol form. The presented model is in good agreement with the experimental results. However, additional experiments with shorter pulses and within a wider wavelength range are required to obtain enough information for understanding the complete mechanism of photoinduced enol $\rightarrow$ keto transformation.

\section{References}

1. P. Markov and I. Petkov, Tetrahedron 33, 1013 (197,7).

2. D. Veierov, T. Bercovici, E. Fischer, Y. Masur and A. Yogev, J. Am. Chem. Soc. 99, 2723 (1977).

3. B. Coucheron, J. Le Saint and P. Courtot, Bul. Soc. Chem. Fr. Ser. II 381 (1980).

4. P. Markov and F. Fratev, C.R. Acad. Bulg. Sci. 28, 771 (1975).

5. G. Nikolov and P. Markov, J. Photochemistry 16, 93 (1981).

6. G. Nikolov and P. Markov, Annuaire de l'Université de Sofia, Faculté de Chimie 72 (1), 109 (1977-1978).

7. S. Savov, S. Saltiel and I. Tomov, Opt. Laser Techn. 13, 193 (1981).

8. P. Markov, J. Photochemistry 6, 91 (1976-1977).

9. F. Fratev, P. Markov and R. Vasileva, Izv. Otd. Khim. Nauk, BAN 7, 737 (1974).

10. G. Kittel, G. Köhler and N. Getoff, J. Phys. Chem. 83, 2174 (1979). 